\title{
Detecting On-Street Parking Spaces in Smart Cities: Performance Evaluation of Fixed and Mobile Sensing Systems
}

\author{
Cristian Roman, Ruizhi Liao, Peter Ball, Member, IEEE, Shumao Ou, Member, IEEE, and Martin de Heaver
}

\begin{abstract}
As the number of vehicles continues to grow, parking spaces are at a premium in city streets. In addition, due to the lack of knowledge about street parking spaces, heuristic circling in the streets not only costs drivers' time and fuel, but also increases city congestion. In the wake of the recent trend to build convenient, green and energy-efficient smart cities, common techniques adopted by high-profile smart parking systems are reviewed, and the performance of the various approaches are compared. A mobile sensing unit has been developed as an alternative to the fixed sensing approach. It is mounted on the passenger side of a car to measure the distance from the vehicle to the nearest roadside obstacle. By extracting parked vehicles' features from the collected trace, a supervised learning algorithm has been developed to estimate roadside parking occupancy. Multiple road tests were conducted around Wheatley (Oxfordshire) and Guildford (Surrey) in the UK. In the case of accurate GPS readings, enhanced by a map matching technique, the accuracy of the system is above $90 \%$. A quantity estimation model is derived to calculate the density of sensing units required to cover urban streets. The estimation is quantitatively compared to a fixed sensing solution. The results show that the mobile sensing approach can perform at the same level as fixed sensing solutions when accurate location information is available but substantially fewer sensors are needed compared to the fixed sensing system.
\end{abstract}

Index Terms -on-street/roadside parking, sonar/ultrasonic, supervised learning, map matching, parking sensors, crowdsourcing

\section{INTRODUCTION}

The continuous growth of vehicles and the waves of urbanization made street parking spaces a premium feature in most cities. The lack of real-time knowledge about the roadside parking occupancy causes vehicles to drive around urban streets searching for an empty parking space. This not only wastes time and fuel, but also increases traffic flows. A downtown traffic study [1] on several major cities reveals that cruising for kerb vacancies is an often overlooked source of congestion, accounting for up to 30 percent of total traffic

Manuscript received May 8, 2017; revised December 1, 2017; accepted January 25, 2018

C. Roman, P. Ball and S. Ou are with the School of Engineering, Computing and Mathematics, Oxford Brookes University, UK (e-mail: cristian.roman,pball,sou@brookes.ac.uk).

R. Liao is with the School of Humanities and Social Science, The Chinese University of Hong Kong (Shenzhen), China (e-mail: rzliao@ cuhk.edu.cn).

M. de Heaver is CEO of GEOmii Technologies Ltd. and Visiting Senior Research Fellow at King's College London, UK (email:martin.deheaver@ethosvo.org).

C. Roman and R. Liao are co-first authors. flows ${ }^{1}$. The frustrating search process, along with the time and fuel wastes are the type of challenges or issues that an Intelligent Transportation System (ITS) aims to address, or broadly speaking, to build convenient, green and energyefficient Smart Cities. There are various ITS or Smart City projects related to parking monitoring with different focus points, such as private parking [2], off-street parking [3] [4] and on-street parking [5] [6].

The first type, private parking projects, originate from the peer-to-peer based sharing economy, where the concept is to rent out owners' spare facilities - e.g. Airbnb for rooms, Uber for cars, and JustPark for empty driveways. In the UK alone, there are an estimated 17 million residential parking spaces such as garages and parkways [7]. The second type, off-street parking, refers to municipal car parks or large fields that can accommodate hundreds of vehicles. The overall occupancy of off-street car parks can be easily monitored by applying entrance counters, acoustic or vision based sensing techniques. Individual bays can also be monitored using fixed sensors in each parking bay which can be used to guide drivers to available spaces. The parking availability can be disseminated via mobile applications (App) or web portals (e.g. Parker and Parkopedia). The third type, on-street or roadside parking, refers to parking spaces along public roads and is the focus of this paper. It accounts for a considerable fraction of urban parking, and can be a convenient option for drivers. However, on-street parking spaces are not usually monitored. The reasons are twofold. Firstly, placing a sensor in each parking space is not a scalable solution when there are a large number of distributed spaces to monitor. In addition, compared to off-street parking, on-street parking has a more challenging environment, (e.g. harsh weather or light conditions) which may impair the sensors' performance (e.g. camera). Other considerations involve road installation, maintenance and municipal coordination issues. In the UK, it is estimated that the number of on-street and off-street public parking spaces to be circa 11 million, but this figure is likely to under-represent the non-regulated parking sector, for which few local authorities have information [7].

The aim of this work is to propose a mobile on-street parking space detection system that is scalable and manageable and to compare its performance with a fixed sensing solution. The mobile detection system employs ultrasonic sensors

\footnotetext{
${ }^{1}$ The surveyed cities include New York, San Francisco, Freiburg, Jerusalem, Cape Town, Sydney and London.
} 
TABLE I: Comparison of On-Street Parking Projects

\begin{tabular}{|c|c|c|c|c|c|c|}
\hline Name & Year & Sensing Technology & Sensor/spot & Accuracy** & Roadworks & Notes \\
\hline ParkNet [8] & 2010 & Sonar & $<1$ & $95 \%$ & No & $\begin{array}{l}\text { Crowdsource sensing. Environmental } \\
\text { fingerpinting to reduce GPS errors }\end{array}$ \\
\hline ParkSense [9] & 2013 & $\begin{array}{l}\text { Mobile Phones } \\
\text { Wi-Fi }\end{array}$ & 1 & $83 \%$ & No & $\begin{array}{l}\text { Use of beacons between mobile phone } \\
\text { and Wi-Fi to infer parking status }\end{array}$ \\
\hline Street Parking System [10] & 2013 & Magnetic & 1 & $98 \%$ & Yes & $\begin{array}{l}82 \text { sensors are deployed at Shenzhen } \\
\text { Institute of Advanced Technology }\end{array}$ \\
\hline SFpark [5] & 2014 & Magnetometer & $>1$ & $86 \%$ & Yes & Complete on/off-street solution \\
\hline Smart Santander [11] & 2014 & Ferromagnetic & 1 & NS & Yes & $\begin{array}{l}\text { Part of Smart City Project } \\
375 \text { sensors were deployed }\end{array}$ \\
\hline FASTPRK [6] & Cnt. & Magnetic & 1 & $95 \%$ & Yes & $\begin{array}{l}\text { Company portfolio including sensing, } \\
\text { analysis, open data interface and App }\end{array}$ \\
\hline GEOmii [12] & Cnt. & Magnetometer & 1 & $86 \%$ & Yes & $\begin{array}{l}\text { Real time and predicted } \\
\text { future parking space availability }\end{array}$ \\
\hline Integrated Smart Parking [13] & Cnt. & Radar & $<1$ & NS & Mount & Also sensing traffic flow \\
\hline Parking Spotter [14] & Cnt. & Sonar/Radar & $<1$ & NS & No & $\begin{array}{l}\text { Crowdsource Sensing } \\
\text { Ford proprietary application }\end{array}$ \\
\hline Smart Parking [15] & Cnt. & $\begin{array}{l}\text { Infrared + } \\
\text { Magnetic }\end{array}$ & NS & NS & Yes & $\begin{array}{l}\text { Company portfolio including sensing, } \\
\text { guiding, payment and management }\end{array}$ \\
\hline
\end{tabular}

mounted on the side of a vehicle to measure the distance from the vehicle to the roadside. A supervised learning algorithm is designed to analyse the structure of the sonar trace to differentiate parked cars from road clutter. Only a reduced set of the sensed data, namely, the interpreted parked vehicles and empty spaces, together with Global Positioning System (GPS) coordinates, vehicle speed and timestamps, can be transmitted to a central server, where a parking occupancy map is built. A novel feature of this approach is that it incorporates a Map Matching (MM) technique to accommodate for any inaccuracy in the received GPS location information. The parking availability information is updated each time a sensor equipped vehicle finds a space. The parking space availability is disseminated to users via a mobile App or a web portal. The system could be developed into a user-engaged crowdsourcing platform where users install the system on their vehicles and contribute their findings to the database. The performance of the mobile system is evaluated by comparing the parking spaces identified by the mobile system to those identified by fixed, on-street parking sensors. Both readings are then validated against a ground truth provided by a camera mounted on the testing vehicle which monitors the spaces as it drives by. An estimation model is also proposed to calculate how many mobile sensing units are needed to cover urban streets with certain updating frequency. The estimation produced by the model is compared to the number of fixed sensors that are needed to cover the same area. The results show that the mobile sensing solution would need a significantly lower number of sensing units than the fixed sensing system.

The rest of the article is organized as follows. First, Section II reviews relevant on-street parking systems, and summarizes representative features of each work. Section III then explains the design and implementation of the proposed mobile sensing system and the supervised learning algorithm. Section IV analyses the initial drive tests in Wheatley, Oxfordshire, and validates the prototype with the visual truth. Section V compares the mobile sensing system with the fixed sensing solution and visual truth in Guildford, Surrey. Section VI estimates the required number of mobile sensing units to cover urban streets. Finally, Section VII concludes the article and introduces several suggestions for further work.

\section{RELATED WORK}

An overview of reported on-street parking systems and their representative features are summarized in this section.

One of the most publicised on-street parking projects is SFpark [5], a U.S. federally-funded parking management project in San Francisco, which spans from 2009 to 2014 with a total budget of 46.2 million dollars. It adopts a wireless sensor network structure, where the data flows from parking sensors and parking meters to a data warehouse via a wireless mesh network. The system gives a complete on/off-street parking solution and provides a range of benefits: easier to find parking (searching time decreases by $43 \%$ ), reduced congestion (traffic volume decreases by $8 \%$ ), lower parking rates (\$11 cents cheaper) and fewer parking tickets (23\% lower). However, the cost of this high-profile pilot deployment parking system is rather high: SFpark installed 11700 magnetometer sensors and 300 pole-mounted mesh nodes for 8000 parking spaces (one or two sensors are installed in each parking space). According to SFpark's finance figures, the parking sensors cost $\$ 5.7$ million 


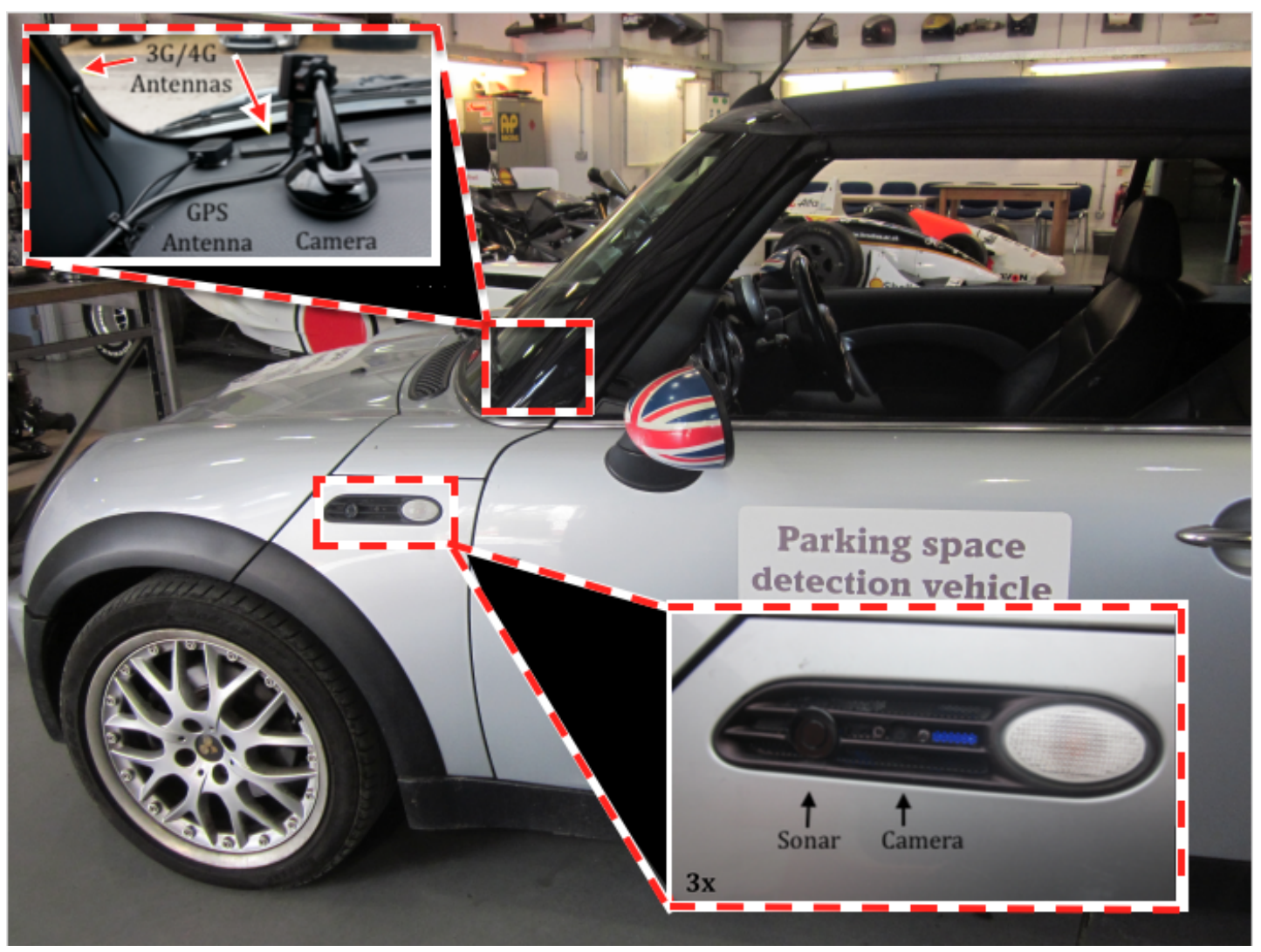

Fig. 1: System prototype depicting the GPS, cellular antennas with the sonar range finder mounted on the BMW Mini passenger side door. The camera was only used for research purposes to validate the system readings.

(approximately $\$ 480$ each), which do not account for management, installation and maintenance expenditure. FASTPRK (magnetic) [6], Street Parking System (magnetic) [10], Smart Santander (ferromagnetic) [16], GEOmii (magnetometer) [12], and Smart Parking (infrared) [15] are similar parking projects using different sensing techniques.

Integrated Smart Parking Solution [13] is a Siemens-led ongoing project aiming at simplifying the searching process, which launched a testing pilot in Berlin in September, 2015. This is different from the previously mentioned embedded sensor approaches as radar sensors are mounted on street lamps to scan larger areas. The radars monitor not only traffic flows but also parking spaces, which can be either used for traffic control or facilitating drivers to find a parking spot. The benefits of the overhead radar approach are claimed to be as follows. Firstly, it is not impaired by weather or light conditions. Secondly, it detects more than just parking spots as it can measure vehicle speed, traffic flows and pedestrian flows. Thirdly, it is mounted on street lamps, which alleviates the infrastructure changes.

Parking Spotter [14] is a joint work by Ford and Georgia Tech. The idea is to leverage sonars and radars that are already widely employed on Ford cars to sense the on-street parking occupancy. The sensing results are updated to a cloud server, and the results are presented to other Ford drivers as an addedvalue service.

Mathur et al. [8] presented ParkNet, a mobile street parking system, which collects parking occupancy information as vehicles pass by. ParkNet consists of a GPS receiver and an ultrasonic rangefinder. Over a one-month trial runs with three vehicles passing by the urban streets of Highland Park, New Jersey, the authors built a parking map from collected data. In order to achieve improved location accuracy, the authors utilize an environmental fingerprinting approach, namely, using objects on the street to correct GPS errors. Mathur et al.'s approach is the closest to the approach taken in this paper but map matching is used instead of the environmental fingerprinting and a new detection algorithm is applied.

Nawaz et al. [9] propose a Wi-Fi beacon association based sensing system named ParkSense to estimate if a driver has entered or driven away from a parking spot. More specifically, ParkSense uses the Wi-Fi association and de-association changing rates to sense the parking status. From the empirical evaluation, the authors claim that the Wi-Fi beacon rate is highly correlated with driver's activity. The disadvantages of the system are that 1) the presence of both mobile phone and Wi-Fi infrastructure are required, and 2) the access to spatially distributed $\mathrm{Wi}-\mathrm{Fi}$ access points for analysis is assumed.

The features of the surveyed parking systems are summarized in Table I. The accuracy of the detection mechanisms depends on the parameters and scenario. If not clearly specified in the available documentation, values have been calculated based on the reported experiments. If no information was made available, they have been marked as NS (Not Specified). 


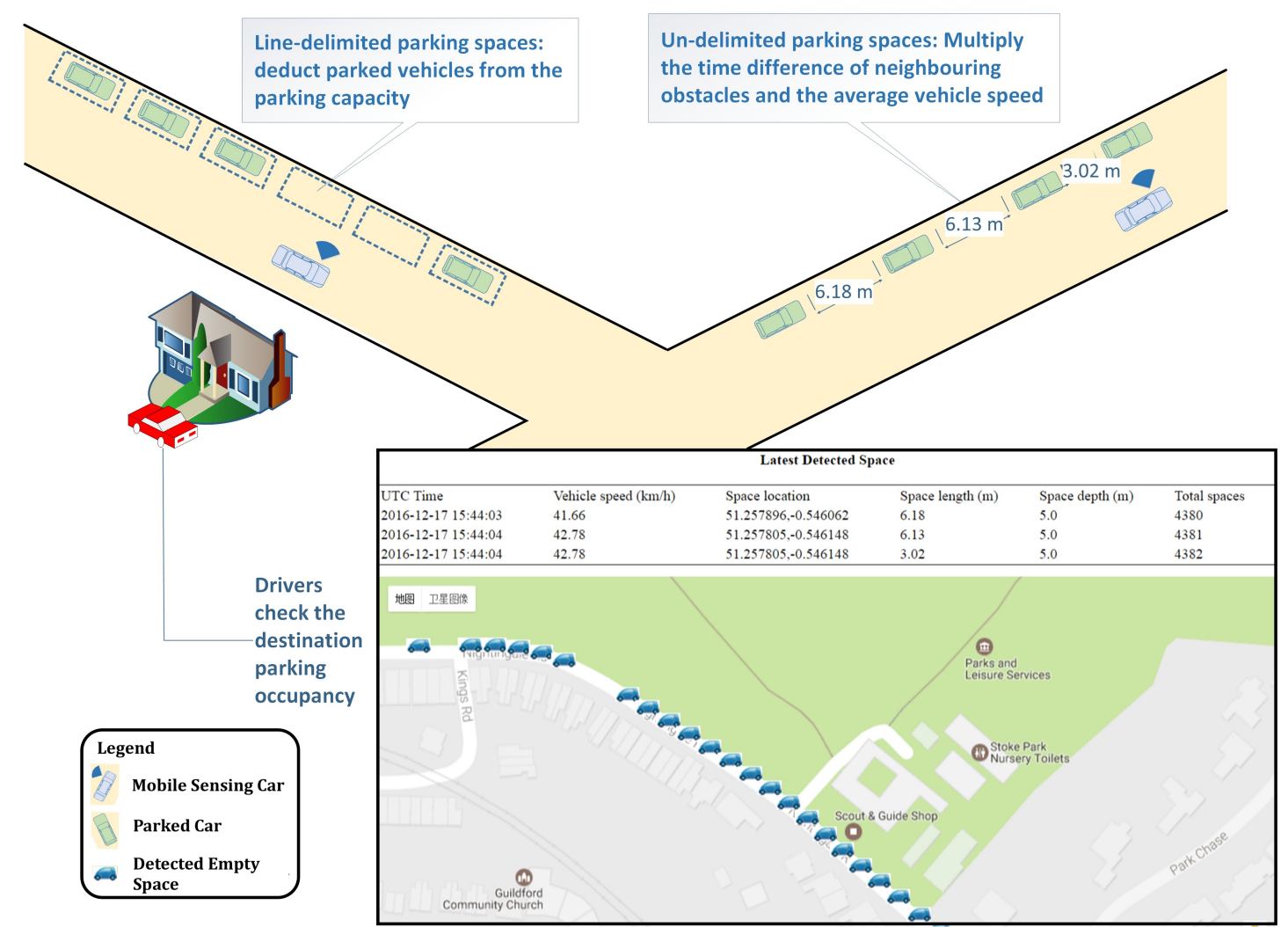

Fig. 2: Scenario description of mobile sensing unit and user web interface to represent detected empty spaces

\section{On-Street Parking Space Detection System: PROTOTYPE, AlgoRithM AND DisSEMINATION OF PARKING INFORMATION}

Firstly, in section III-A the prototype system is described. In section III-B the techniques of the supervised learning algorithm are discussed. Section III-C presents the limitations of the system and finally, in section III-D, the presentation method of the detected vehicles and empty spaces are outlined.

\section{A. The Prototype System and Architecture}

The prototype kit consists of an ultrasonic rangefinder, installed on the passenger side of the vehicle, and a Raspberry $\mathrm{Pi}$ with a GPS receiver and $3 \mathrm{G} / 4 \mathrm{G}$ connection placed in the glove compartment of the BMW Mini (Fig. 1). It is to be noted that the video camera next to the GPS is not part of the mobile sensing system. Its purpose is to record the ground truth which can be used to validate the sensor measurements.

The ultrasonic rangefinder is set to transmit a short pulse every 50 milliseconds. The emitted wave is reflected when there is an object in the way. By counting the elapsed time, together with the sound speed in the air, the distance to the object is measured. The measurement data is locally preprocessed to reduce the data traffic. Only data that is interpreted as parked vehicles or empty spaces by the algorithm are sent to the central database.

The on-board communication links or a cellular $(3 \mathrm{G} / 4 \mathrm{G})$ connection can be used for the back-haul data transmission. A software bundle consisting of Linux, Apache, MySQL, and PHP (LAMP) is employed to present the data outputted by the algorithm on the mobile sensing system. The central database includes information on the available parking zones (i.e. the capacity and GPS coordinates of parking zones such as the red areas in Fig. 6), which are used to identify empty parking spaces from the received sonar data. The software bundle identifies valid parking spaces and the inferred results are accessed via mobile Apps or web portals by users (Fig. 2).

\section{B. The Supervised Learning Algorithm}

A supervised learning algorithm is developed to recognise parked cars and empty spaces. Contouring parameters are extracted from the drive-test trace to train a classifier in discriminating parked cars and spaces from road clutter.

A series of initial drive tests were conducted on two roads in Wheatley, Oxfordshire. In the tests, two streets were repeatedly circled to observe the repeatability of the outputted results and to emulate the possible update frequency of a crowdsourcing approach. As shown in Fig. 3, High Street (Route1) has two car parks (park1 has 6 spaces and park2 has 1 space), and Church Road (Route2) has three car parks (park3 has 1 space, park4 has 11 spaces and park5 has 5 spaces).

Fig. 4 shows a sonar reading plot as the testing vehicle passes by Route1. As can be seen from the figure, the sonar reading present a series of detected objects which include seven parked vehicles ( 6 in park 1 and 1 in park2) and 2 street side poles in the form of distinctive U-shaped contours. Therefore, the sonar data can be interpreted to a fully occupied park1 and park2 of Route1. The contouring features also allow us to distinguish parked vehicles from road furnitures (e.g. 


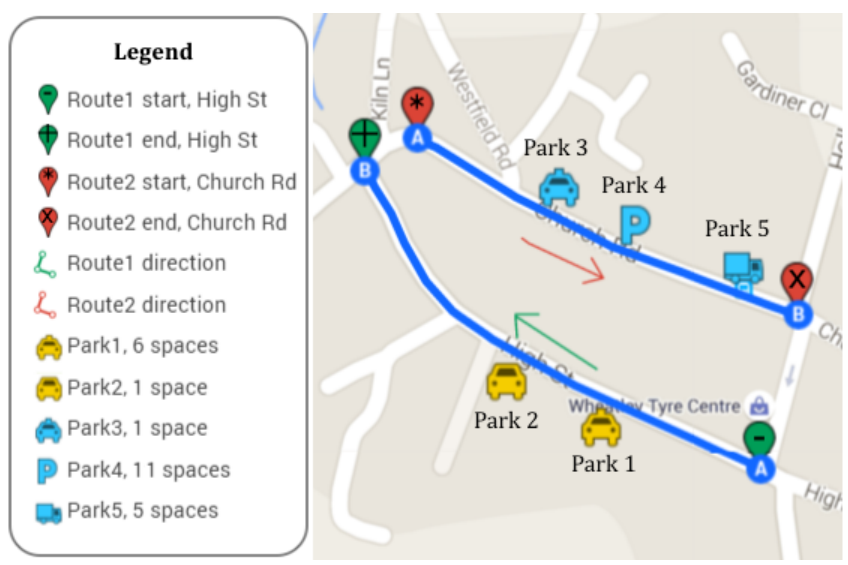

Fig. 3: Drive test route in Wheatley, UK along High Street and Church Road

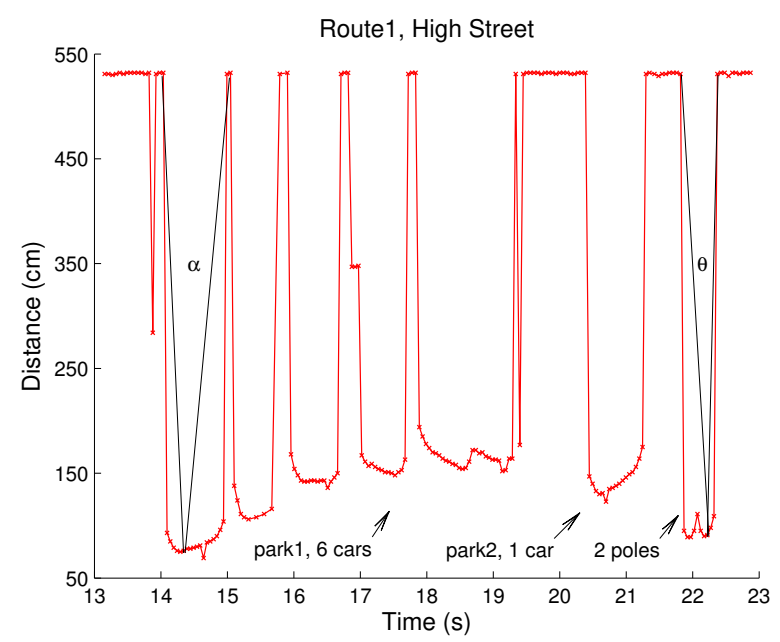

Fig. 4: Trace plot of fully parked Route1 (compressed image; $\alpha$ and $\theta$ not to scale)

the two poles on the right of Fig. 4). The classifier training parameters are summarized as follows:

1) Distance: From the monitoring vehicle perspective, it is assumed that the distance to the parked cars is between $70 \mathrm{~cm}$ and $250 \mathrm{~cm}$. The lower bound of the range corresponds to lateral safety distance or minimum safety distance (Distance $\left._{\min }=70 \mathrm{~cm}\right)$. The higher bound of the range (Distance $\max =250 \mathrm{~cm}$ ) is obtained by adding a car's width, assuming the maximum width is $180 \mathrm{~cm}$.

2) Length: 2.1 meters is adopted as a minimum vehicle length $\left(\right.$ Lengt $\left._{\min }\right)$ to categorize the trace for calculating the 'small-scale' standard deviation, and 9 meters as a upper bound (Length $h_{\max }$ ) for deriving the 'large-scale' standard deviation.

3) Standard Deviation: The distance range filters out most of the road clutter that is outside $\left[\right.$ Distance $_{\min }$, Distance $\left._{\max }\right]$, but some still remain. Standard deviation of the signal variation within a minimum, maximum Range is used to identify the signature of a vehicle. The Range is normalised from the car speed and length parameter $\left(\right.$ Length $_{\min }$, Length $\left._{\max }\right)$ in the time domain. The vehicles' core frame corresponds to the relatively flat $\mathrm{U}$-shaped bottom for which the standard deviation is small, also referred to as the small-scale standard

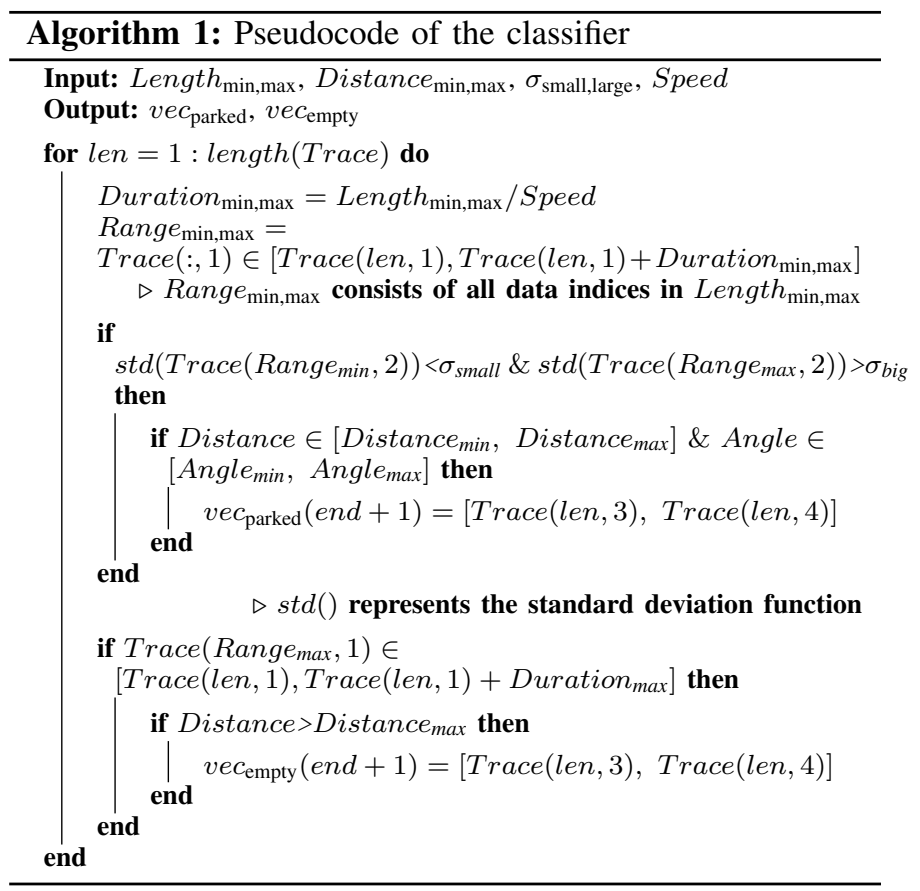

deviation $\left(\sigma_{\text {small }}\right)$. The standard deviation at the edge of the U-shaped bottom is larger, and can be used to describe the vehicles' edges, also referred to as the large-scale standard deviation $\left(\sigma_{\text {large }}\right)$. Based on the trace of the drive tests we conducted in Wheatley, $\sigma_{\text {small }}$ and $\sigma_{\text {large }}$ have empirical values of 10.9 and 51.3 respectively.

4) Angle: The angle between the vertices and the bottom of the detected object is calculated using trigonometric functions ( $\alpha$ in Fig 4). Based on the trace of the drive tests we conducted in Wheatley, the angle of the contour of parked vehicles ranges from $80^{\circ}$ to $130^{\circ}$. This contour angle range is applied in the classifier to mask out road furnitures. It is to be noted that, the angle range $[80,130]$ only applies in the drive tests with an average speed of $20 \mathrm{~km} / \mathrm{h}$. In more general cases, the angle range needs to be normalised with different speeds.

These four contouring parameters (distance, length, standard deviation, and angle) allow us to find as precise as possible the pattern of a parked vehicle or space. For example, consecutive poles on the roadside are discarded by measuring the narrow angles between the posts ( $\theta$ in Fig. 4). However, the angle alone may not prove to be adequate in all situations. For instance, to distinguish both cars 5 and 6 from park1 (Fig. 4 ), the angle of the contour gives an indication that a single car is detected. The standard deviation identifies two edges (i.e. a single car), respectively start of car 5 and end of car 6 . The distance also indicates that a single car is present since there is no space in between the cars. However, the length of the detected trace, derived from the speed, indicates that there are two cars parked rather than one. The combination of all four contouring parameters are thus needed to correctly identify cars and spaces. The four contouring parameters form a feature vector, which is used to train the classifier. The classifier interprets the fed data into groups of road clutter, parked vehicles or empty spaces and the results are used for the next iteration of the algorithm. 
As illustrated in Algorithm 1, the classifier iterates through the gathered Trace. For each iteration, the algorithm first uses the vehicle length range $\left(\right.$ Lengt $\left._{\min , \max }\right)$ divided by the speed to identify all the indices of trace $\left(\right.$ Range $\left._{\min , \max }\right)$, corresponding to the length range. The data is then checked to see if it matches the above-mentioned patterns (e.g. the standard deviation of the U-shaped trough contour $\left(\sigma_{\text {small,big }}\right)$, the lateral distance range $\left(\right.$ Distance $\left._{\min , \max }\right)$, and the contouring angle range $\left(\right.$ Angle $\left._{\min , \max }\right)$. The classifier will output the results into the parked vehicles vector $\left(v e c_{\text {parked }}\right)$ or the empty spaces vector ( $\left.v e c_{\text {empty }}\right)$.

\section{Limitations of mobile sensing approach}

Some of the limitations of the current mobile sensing system are lane-changing detection and density of monitoring vehicles.

1) Lane-changing detection: The designed algorithm and current sonar configurations can only handle the single lane situation. If the vehicle moves to a second lane, then the sonar may misunderstand the extra distance as empty parking spaces. One solution is to employ a high-precision GPS. However, it may be difficult as the adjacent lanes are within just a few meters. Another solution to detect lane-change could be to use two ranger sensors positioned at each end of the car and measure the differential signal [17]. For example, if the vehicle moves away from one lane to another, then the difference of the two sensors' reading remains constant if we adjust the lateral time difference. Other scenarios where the current system may give inaccurate results are perpendicular parking or cycle lanes with a cyclist in between the vehicle and the parking bays.

2) Density of monitoring vehicles: Using the mobile sensing system, the parking data update rate will depend on how often a sensing vehicle passes a given parking bay. A driver may get to an advertised space and arrive to find it is occupied. One way to improve the currency of the data is to provide future occupancy prediction based on open data or history data [18]. Another approach is to design a reservation system or a balancing algorithm that guides people to different locations if multiple requests to the same space are received. Such a system is only appropriate if there is a smart meter deployed for each bay, as used in some parts of the United States. Enforcement would still have to be performed via parking agents circling the areas.

\section{Visualisation of Empty Spaces and Detected Vehicles}

The empty spaces can be presented in two ways: either as a generic 'space' or a space with variable length. The former is derived using a predefined length threshold or deducting parked vehicles from the parking capacity, and is suitable for line-delimited parking spaces; while the latter is adaptively obtained by multiplying the time difference of two neighbouring obstacles and the average speed, which is suitable for un-delimited spaces and flexible for compact cars or long lorries. Fig. 2 depicts both options.

These options can be further developed to include disabled parking bays, bus stops, car clubs or delivery only bays.
Tags to represent these specific options can be included when the database is populated with the valid parking spaces. The appropriate tag can be applied after querying the database when a 'generic' space is received by the server.

Detecting parking violations can also be performed by checking whether GPS coordinates of the detected cars are in valid parking bays. If the cars are outside the permitted parking zones, they can be marked on the map as illegal parked cars or posing a safety risk. However, using the system for parking enforcement may deters the users from participating in the data collection.

\section{Validation of Sonar Based Mobile Sensing SYSTEM}

Initial tests have been carried out in Wheatley, Oxfordshire, to assess the ability of the sonar sensor system to detect vehicles and spaces in the presence of roadside clutter. The accuracy of the vehicle and space detection was based on local assessment of the parking occupancy.

The average cruising speed in the drive test was $20 \mathrm{~km} / \mathrm{h}$, and the circuit time was 120 seconds, thus, the vehicle passed by the same point every 2 minutes. As the on-street parking zones on High St and Church Rd are un-delimited parking, the length and the parking manner of the parked cars heavily affect the detection results. Therefore, it may not be accurate to infer the number of parking spaces by subtracting the number of detected cars from the parking capacity. For this reason, parking spaces are given in two ways: 1) the number of parking spaces by subtracting the number of detected cars from the parking capacity; 2) parking spaces with detected available length.

\section{A. Parked Vehicle Detection}

Table II gives the statistics of parked vehicles from park1 to park5 with 6 runs. The $D V / G T / P C$ data format denotes the number of detected vehicles/ground truth/parking capacity. For example, in the fifth run of park1 (Table II), there were three cars detected by the algorithm (system detection), three cars actually parked (ground truth), and six parking spaces in total (parking capacity). The last column shows the false positives of each run, which is where road clutter has been detected as a vehicle but since the detections occurred outside known parking bays, it does not affect the accuracy.

The ratio of sensed vehicles divided by the actual number of vehicles in parking bays is $123 / 124=99.2 \%$. The accuracy can increase if the updating frequency is higher or if there are more mobile sensing units employed, the relationship of which is explored in Section VI and Fig. 11.

\section{B. Space Detection}

In Table III, in the data format $D S / D S L / G T, D S$ denotes the number of detected spaces by subtracting from parking capacity, DSL denotes the length of the detected spaces and $G T$ is the visual ground truth of un-occupied bays. For example, in the fifth run of park1, 3 spaces are inferred by the subtraction of detected vehicles, 2 spaces with different length 


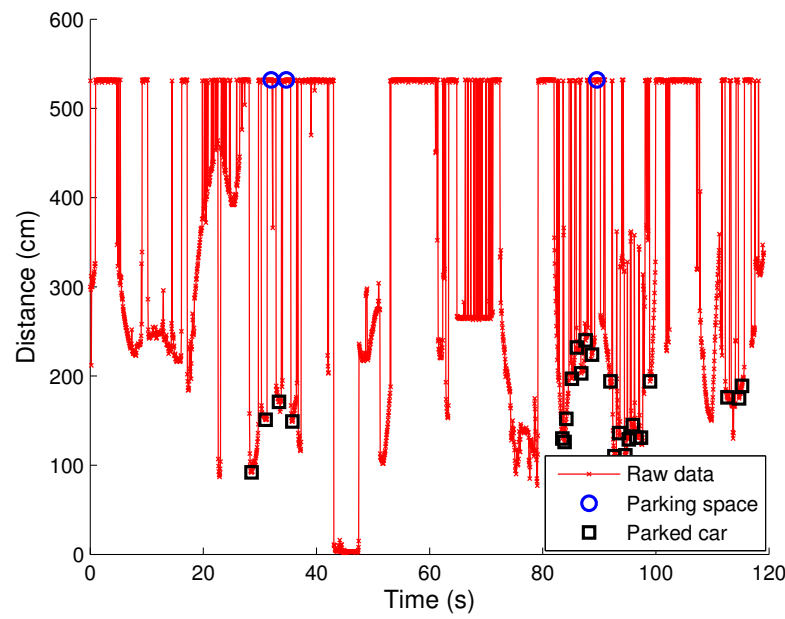

(a)

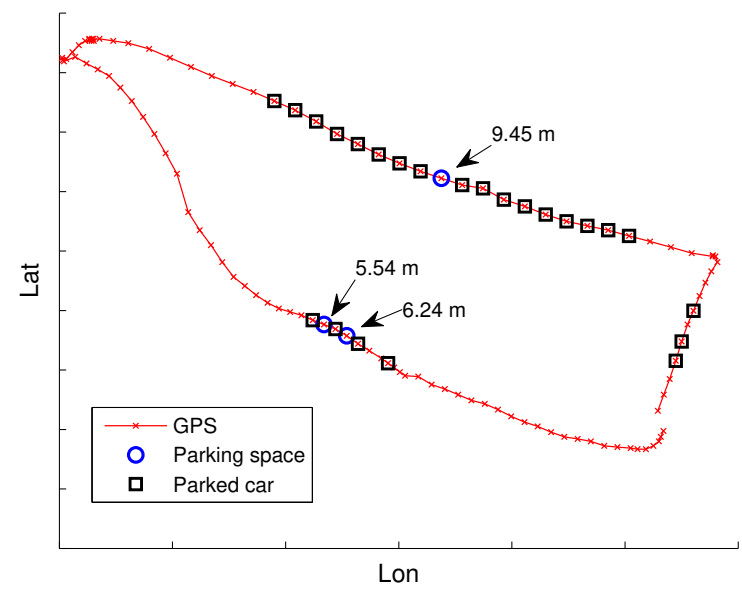

(b)

Fig. 5: Trace plot of Run 5 showing the sensor raw data (a) and the space map of the run (b).

TABLE II: Parked Vehicle Detection - Results in Wheatley

\begin{tabular}{|c|c|c|c|c|c|c|}
\hline Run \# & $\begin{array}{c}\text { park1 } \\
\text { DV/GT/PC }\end{array}$ & $\begin{array}{c}\text { park2 } \\
\text { DV/GT/PC }\end{array}$ & $\begin{array}{c}\text { park3 } \\
\text { DV/GT/PC }\end{array}$ & $\begin{array}{c}\text { park4 } \\
\text { DV/GT/PC }\end{array}$ & $\begin{array}{c}\text { park5 } \\
\text { DV/GT/PC }\end{array}$ & False Positives \\
\hline 1 & $6 / 6 / 6$ & $1 / 1 / 1$ & $1 / 1 / 1$ & $10 / 10 / 11$ & $5 / 5 / 5$ & 2 \\
\hline 2 & $6 / 6 / 6$ & $1 / 1 / 1$ & $1 / 1 / 1$ & $10 / 10 / 11$ & $5 / 5 / 5$ & 4 \\
\hline 3 & $6 / 6 / 6$ & $0 / 0 / 1$ & $1 / 1 / 1$ & $10 / 10 / 11$ & $5 / 5 / 5$ & 3 \\
\hline 4 & $2 / 2 / 6$ & $0 / 0 / 1$ & $1 / 1 / 1$ & $10 / 10 / 11$ & $5 / 5 / 5$ & 3 \\
\hline 5 & $3 / 3 / 6$ & $1 / 1 / 1$ & $1 / 1 / 1$ & $9 / 10 / 11$ & $5 / 5 / 5$ & 2 \\
\hline 6 & $4 / 4 / 6$ & $1 / 1 / 1$ & $1 / 1 / 1$ & $7 / 7 / 11$ & $5 / 5 / 5$ & 2 \\
\hline
\end{tabular}

*- DV: Number of Detected Vehicles / GT: Ground Truth / PC: Parking Capacity

TABLE III: Space Detection - Results in Wheatley

\begin{tabular}{|c|c|c|c|c|c|}
\hline Run \# & $\begin{array}{c}\text { park1 } \\
\text { DS/DSL/GT }\end{array}$ & $\begin{array}{c}\text { park2 } \\
\text { DS/DSL/GT }\end{array}$ & $\begin{array}{c}\text { park3 } \\
\text { DS/DSL/GT }\end{array}$ & $\begin{array}{c}\text { park4 } \\
\text { DS/DSL/GT }\end{array}$ & $\begin{array}{c}\text { park5 } \\
\text { DS/DSL/GT }\end{array}$ \\
\hline 1 & $0 / 0 / 0$ & $0 / 0 / 0$ & $0 / 0 / 0$ & $1 / 6.86 \mathrm{~m} / 1$ & $0 / 0 / 0$ \\
\hline 2 & $0 / 0 / 0$ & $0 / 0 / 0$ & $0 / 0 / 0$ & $1 / 6.56 \mathrm{~m} / 1$ & $0 / 0 / 0$ \\
\hline 3 & $0 / 0 / 0$ & $1 / 7.00 \mathrm{~m} / 1$ & $0 / 0 / 0$ & $1 / 7.23 \mathrm{~m} / 1$ & $0 / 0 / 0$ \\
\hline 4 & $4 / 9.39 m, 9.04 m />1$ & $1 / 9.62 m / 1$ & $0 / 0 / 0$ & $1 / 6.11 \mathrm{~m} / 1$ & $0 / 0 / 0$ \\
\hline 5 & $3 / 6.24 m, 5.54 m />1$ & $0 / 0 / 0$ & $0 / 0 / 0$ & $2 / 9.45 \mathrm{~m} />1$ & $0 / 0 / 0$ \\
\hline 6 & $2 / 6.52 m, 7.09 m />1$ & $0 / 0 / 0$ & $0 / 0 / 0$ & $4 / 7.19 m />1$ & $0 / 0 / 0$ \\
\hline
\end{tabular}

*- DS: Deducted Parking Capacity (Spaces) from Detected Vehicles = PC - DV

**. DSL: Detected Spaces Length / GT: Ground Truth 


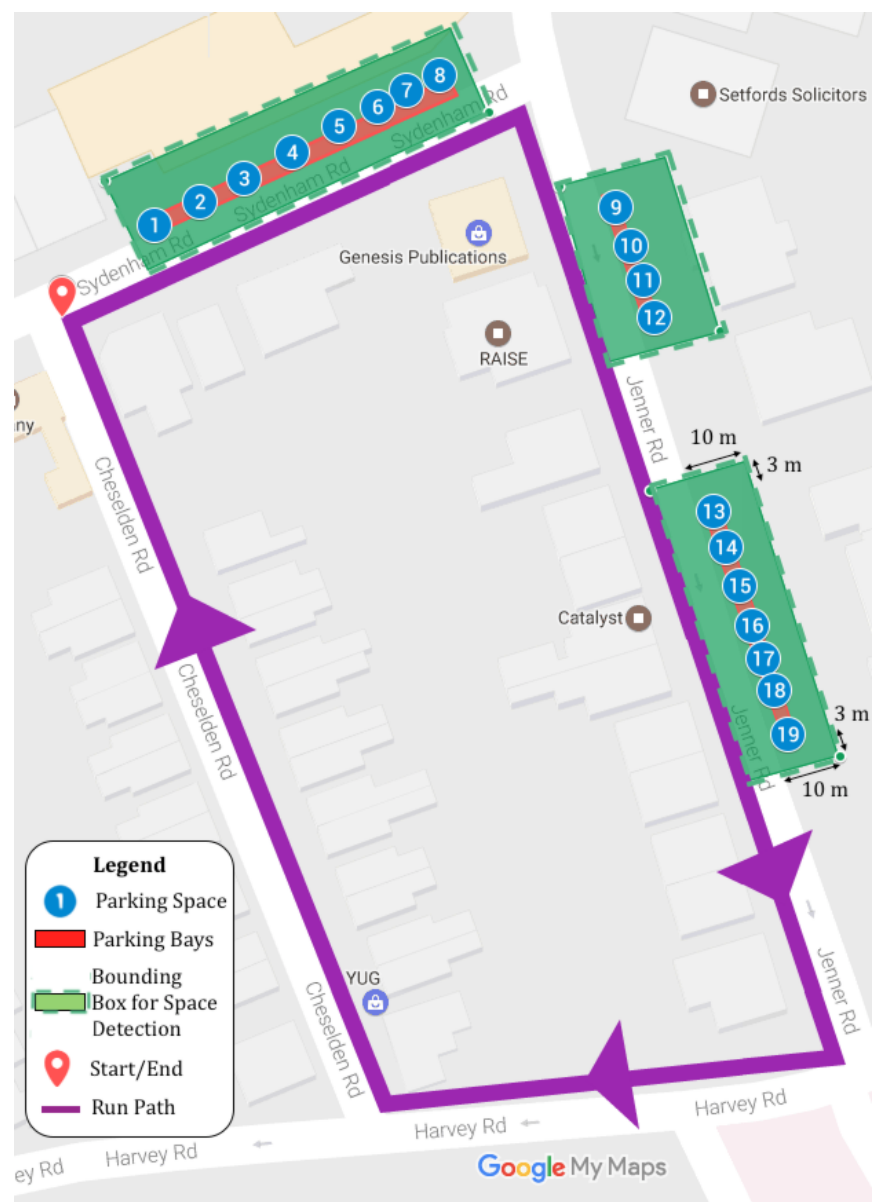

Fig. 6: Parking Locations with sensing equipment in Sydenham Road and Jenner Road, Guildford, UK.

are obtained from the length space detection algorithm, and more than 1 space is observed in the recorded video.

The trace plot of Run 5 and its space map are shown in Fig. 5. The space map in Fig. 5(b), corresponding to the map in Fig. 3, provides the parking space with extra length information, which allows users to identify spaces that match specific vehicle lengths. For example, a small city vehicle can fit in a 3-meter space, while a large vehicle may require a 6-meter space.

\section{Fixed Sensing versus Mobile Sensing: COMPARING THE TWO APPROACHES}

In this section, the mobile sensing system installed in the BMW Mini is evaluated by comparing the parking space detection accuracy obtained via the mobile sensing system with the information provided by the fixed sensors installed in each parking bay. The human analysis of the videos captured by the on-board cameras were used to provide ground truth.

\section{A. Testing Scenario}

As part of a Smart City Scale Demonstrator project, GEOmii Technologies have installed 298 on-street parking sensors on the main shopping streets in Guildford, Surrey [12]. The aim is to monitor car parking spaces and shopper

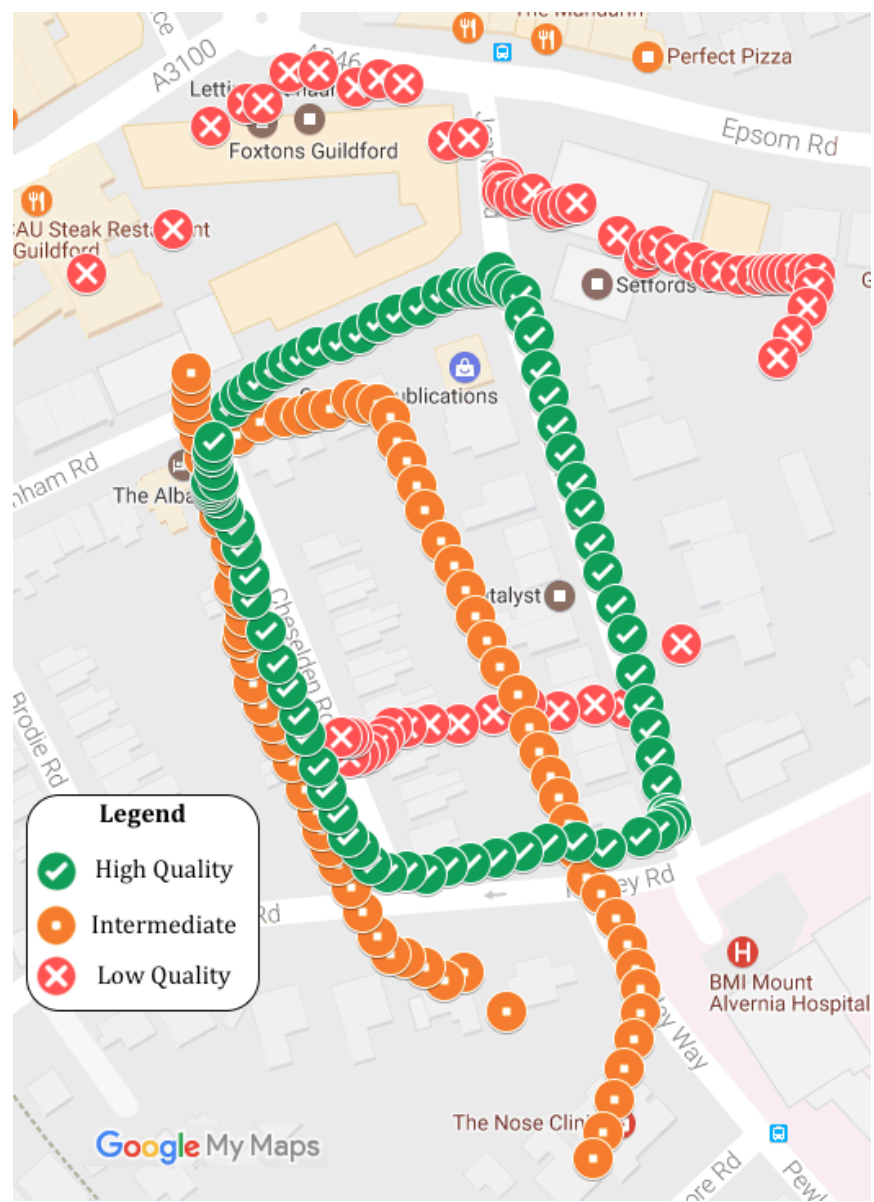

Fig. 7: Example of the three types of GPS Quality Assessment for Guildford Runs $11(\mathrm{~L}), 12(\mathrm{H})$ and $15(\mathrm{I})$.

movements around the town centre. This data will be used by a range of applications that allow shoppers to easily find available parking spaces, retailers to gain valuable insights and the local authorities to better understand Guildford's needs.

The locations chosen for comparing the two parking detection approaches are the streets of Sydenham Road and Jenner Road, close to the High Street shopping street. 8 parking bays are located on Sydenham Road and 11 on Jenner Road, adding up to a total of 19 parking spaces (Fig. 6). Fixed sensors are installed on each of these parking bays and the spaces detected by the mobile sensor are compared with the readings from these fixed sensors.

In total, 22 runs were conducted over the course of a day.The start and end point of a run is the intersection between Cheselden Road and Sydenham Road, as depicted in Fig. 6. The average cruising speed is $16.75 \mathrm{~km} / \mathrm{h}$, and the lap time is around 90 seconds. 11 runs were conducted in the morning (11:00 to 12:30) and 11 runs in the afternoon (13:30 - 15:00).

All the resources consisting of raw readings of drive tests, map representations, along with the recorded ground truth videos have been made available ${ }^{2}$.

\footnotetext{
${ }^{2}$ http://goo.gl/ZU8GA8
} 


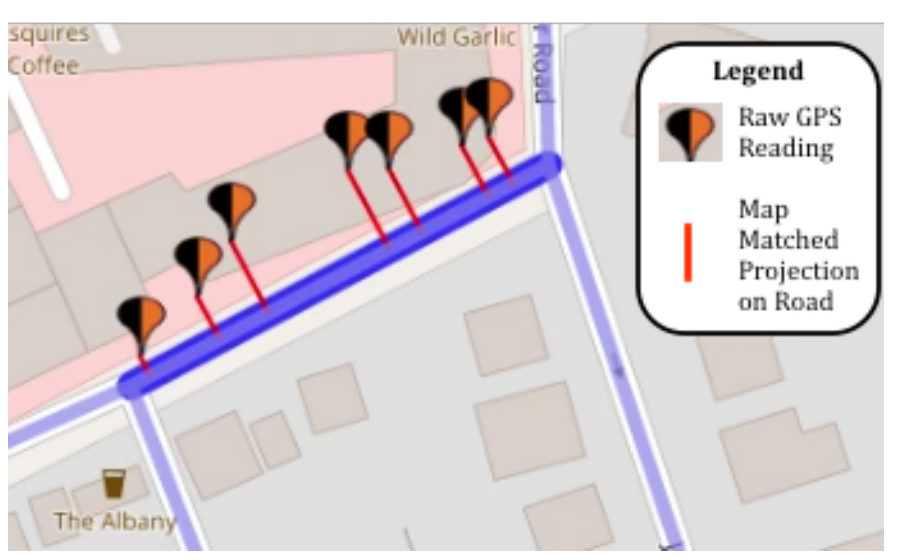

Fig. 8: Map Matching Example of Detected Spaces on Sydenham Road, Guildford, using TrackMatching software.

\section{B. Parking Spaces detected by Fixed Sensing System}

The fixed sensors under the parking bays are connected in a mesh network and update their state every 5 minutes. The sensors are in sleep mode in between two readings in order to save battery life. Due to this fixed timing in reporting information, if there is a change in parking state in between two readings, the reading can be incorrect.

\section{Parking Spaces detected by Mobile Sensing System}

The bounding boxes around each parking bay have been set to $10 \mathrm{~m}$ from the kerb, $3 \mathrm{~m}$ before and after the parking bays, as depicted in Fig. 6 . The $10 \mathrm{~m}$ perpendicular to the road is to allow for GPS error/drift. Any space reported in that bounding box will be marked as a valid parking space.

To identify the parking vehicles or empty spaces, the system relies on the GPS location information. However, standalone GPS may have inaccuracies for the following reasons: atmospheric propagation conditions, number of visible satellites (urban canyon effect) and reflections. One of the solutions, used in the system is to use the map matching technique. Map matching tries to match recorded geographic coordinates to a logical model of the real world (e.g. vehicle localisation above houses will be placed on the adjacent roads). Map matching is the technique used to correct the GPS readings and improve the accuracy of aligning the detected space with the bounding boxes. An example, using the TrackMatching software [19], is depicted in Fig. 8. Currently this is an offline technique but an online real-time version could be developed using TrackMatching or the open source map matching solution provided by GraphHopper, based on the work by Newson et al. [20].

Aside from the post-processing Map Matching technique, Real Time Kinematic (RTK) GPS or Differential GPS (DGPS) could be solutions that can bring the location accuracy below $1 \mathrm{~m}$ and improve the space detection system. However, the current cost of RTK makes this an expensive solution. The new Galileo GPS system could prove to be a more cost-effective solution.

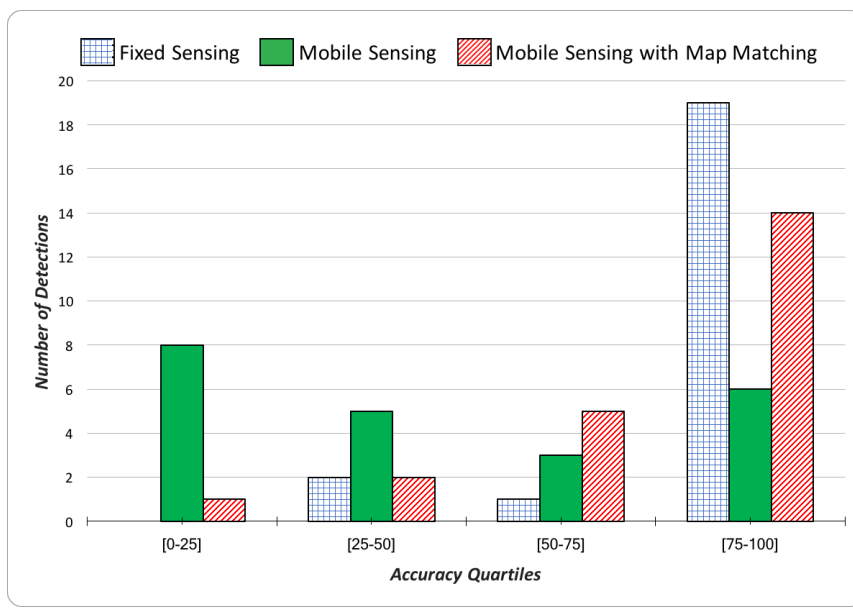

Fig. 9: Number of Detections for each Accuracy Quartile for each of the space sensing technologies for all 22 Runs.

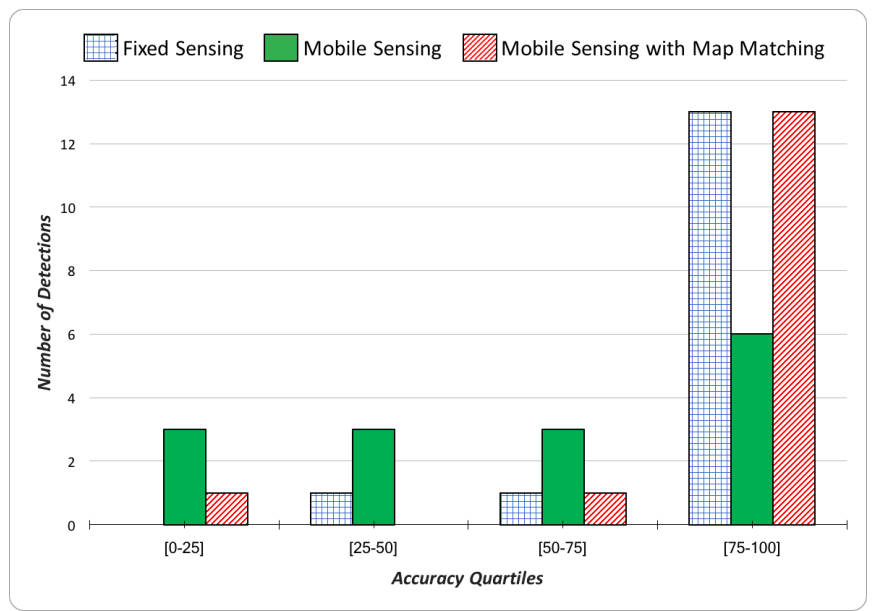

Fig. 10: Number of Detections for each Accuracy Quartile for each of the space sensing technologies for all runs without runs 5 to 11 , corresponding to the low quality GPS readings.

\section{Results Discussion}

The full results are presented in Table IV. A color coding with symbol association is used to have an easier overview of the results. All the GPS quality or accuracy percentages above $75 \%$ are labelled with a green check-mark and marked as High $(\mathrm{H})$. Values between $50 \%$ and $75 \%$ are labelled in gray and marked as Intermediate (I), and values below 50\% are labelled with a red cross and marked as Low (L).

The 'GPS Quality' reported in column 1 is a subjective quality assessment based on the localizations plotted on a map, as shown in Fig. 7. In the case of a noisy and sparse localization distribution of the reported positions, the quality has been marked as low (L) (Red in Table IV). Different weather conditions were noticed in the two testing periods (morning, afternoon) which is one of the reasons that could explain the difference in the quality of GPS readings between morning and afternoon.

It can be observed that the fixed sensing approach is more consistent over the course of the 22 runs than the mobile sensing system. The fixed sensors are not performing well for 
TABLE IV: Comparison table of the various parking space detection approaches

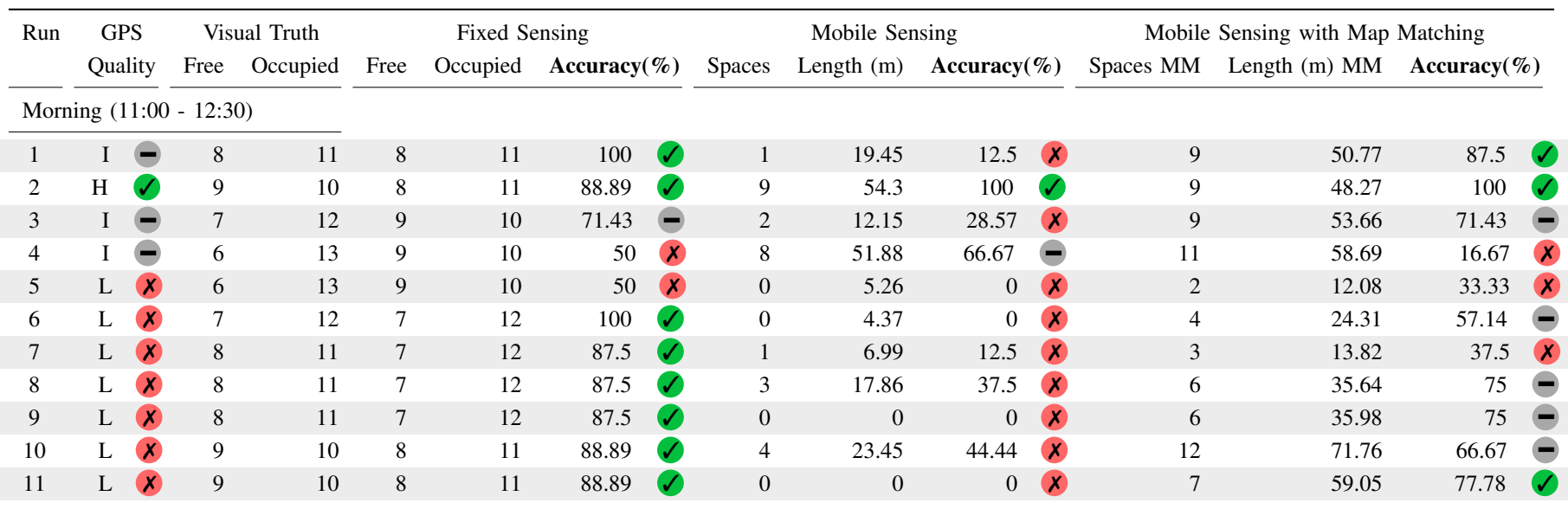

Afternoon (13:30 - 15:00)

\begin{tabular}{|c|c|c|c|c|c|c|c|c|c|c|c|c|c|c|c|c|}
\hline 12 & $\mathrm{H}$ & ( & 12 & 7 & 12 & 7 & 100 & 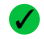 & 13 & 77.29 & 91.67 & 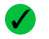 & 13 & 74.76 & 91.67 & D \\
\hline 13 & $\mathrm{H}$ & 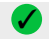 & 12 & 7 & 12 & 7 & 100 & ( & 14 & 81 & 83.33 & ( & 13 & 72.36 & 91.67 & D \\
\hline 14 & $\mathrm{H}$ & 0 & 15 & 4 & 12 & 7 & 80 & ( & 12 & 75.52 & 80 & ( & 13 & 75.52 & 86.67 & $D$ \\
\hline 15 & I & - & 14 & 5 & 15 & 4 & 92.86 & ( & 0 & 6.07 & 0 & $x$ & 12 & 70.64 & 85.71 & 8 \\
\hline 16 & I & $\theta$ & 13 & 6 & 15 & 4 & 84.62 & 0 & 4 & 24.03 & 30.77 & $x$ & 15 & 90.54 & 84.62 & 8 \\
\hline 17 & $\mathrm{H}$ & 0 & 13 & 6 & 11 & 8 & 84.62 & 0 & 9 & 58.5 & 69.23 & - & 13 & 76.48 & 100 & 8 \\
\hline 18 & I & $\theta$ & 13 & 6 & 11 & 8 & 84.62 & 0 & 11 & 65.08 & 84.62 & 0 & 15 & 86.98 & 84.62 & ( \\
\hline 19 & I & $\theta$ & 13 & 6 & 11 & 8 & 84.62 & 0 & 3 & 21.79 & 23.08 & $x$ & 16 & 92.37 & 76.92 & ( \\
\hline 20 & I & - & 13 & 6 & 12 & 7 & 92.31 & 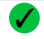 & 6 & 35.98 & 46.15 & $x$ & 13 & 74.37 & 100 & $\checkmark$ \\
\hline 21 & I & - & 13 & 6 & 12 & 7 & 92.31 & ( & 12 & 67.79 & 92.31 & 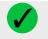 & 13 & 74.05 & 100 & $\sqrt{2}$ \\
\hline 22 & I & - & 13 & 6 & 12 & 7 & 92.31 & 8 & 9 & 54.7 & 69.23 & - & 12 & 65.04 & 92.31 & $\checkmark$ \\
\hline \multicolumn{6}{|c|}{ Average } & & 85.86 & 8 & & & 44.21 & $x$ & & & 76.92 & 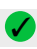 \\
\hline \multicolumn{6}{|c|}{ No Low Quality GPS } & & 85.86 & 8 & & & 54.54 & $x$ & & & 84.65 & 2 \\
\hline \multicolumn{6}{|c|}{ Only High Quality GPS } & & 85.86 & 8 & & & 84.85 & 0 & & & 94 & 2 \\
\hline
\end{tabular}

Legend: $\checkmark$ - High Quality (>75\%); $\quad-$ Intermediate Quality $(>50 \%, \leq 75 \%) ; \quad \chi \quad$ - Low Quality $(<50 \%)$

Runs 3 to 5 when there is a large change in the number of parked cars in a short interval of time. This is not a limitation of the detection technology but it is related to the refresh rate of the parking bay dissemination status.

The mobile sensing system can identify parked cars or empty spaces accurately. However, due to the unstable GPS readings in cities, the detected results are erroneous when presented on maps (44\% of correctly detected spaces). If the map matching technique is applied, the accuracy of the mobile sensor system increases to an average of $76.92 \%$, and even $94 \%$ when the quality of the GPS is high. This may be due to the offset between the parking bays and the GPS readings. When intermediate or high quality GPS localizations are reported, the fixed sensing and mobile sensing system approaches perform equally well.

The combined histograms of the different technologies in Fig. 9 shows that the fixed sensors have a higher count for accuracies above the $75 \%$ threshold. However, when the localization is of intermediate or high quality, both the fixed sensors and the mobile sensing system with map matching perform equally well, with a similar number of counts for the 75-100\% interval (Fig. 10).

The system could monitor the confidence level of the GPS reading, possibly based on the number of satellites that are visible while doing the measurement, and discard any readings that are made with a GPS below a certain quality level.

\section{Estimating Mobile Sensing Units Needed for A CITY}

In this section, the number of mobile sensing systems that are required as a function of the refresh time is calculated. It is envisioned that the mobile sensing kits could be employed on public vehicles such as buses and taxis to emulate the crowdsourcing concept, as they continually scan the city and gather data as they travel along their routes.

Let $s, p$ and $w$ denote the area of a city, the ratio that total roads account for a city area, and the average road width, respectively. The total road length of the city can be obtained: $L=s \cdot p / w$. If the parking occupancy map updating frequency and the vehicle detection accuracy rate are $\tau$ and $\gamma$, the number of mobile sensing units $m$ needed to cover the city can be estimated:

$$
m=\frac{L}{\nu \cdot \frac{1}{\tau} \cdot \gamma},
$$

where $\nu$ is the average cruising speed of mobile sensing vehicles. 
In order to compare with fixed sensing techniques, we use this model to estimate the number of mobile sensing units. San Francisco was chosen as a city because the number of sensors deployed to cover the city are known from the SFpark pilot area: 12000 sensors for 8000 parking spaces. The upper east part of San Francisco has an area of $19.26 \mathrm{~km}^{2}$, where the SFpark pilot is deployed (excluding the metropolitan area) Assuming urban streets account for around $10 \%$ of the city area, and the average street width in San Francisco is 10 meters, the total road length is then derived as:

$$
L=\frac{19.26 \cdot 10^{6} \cdot 10 \%}{10}=1.926 \cdot 10^{5} \text { meters } .
$$

If the parking occupancy map is updated every $5 \mathrm{~min}$ utes (i.e. $\tau=0.0083$ ), equivalent to the values used in the Guildford trials, the vehicle detection accuracy rate is $\gamma=87.9 \%$, and the average city cruising speed is assumed to be $\nu=20 \mathrm{~km} / \mathrm{h}$ (i.e. $5.55 \mathrm{~m} / \mathrm{s}$ ), the required number of mobile sensing units to be mounted on vehicles is:

$$
m=\frac{1.926 \cdot 10^{5}}{5.55 \cdot 300 \cdot 87.9 \%}=131.2
$$

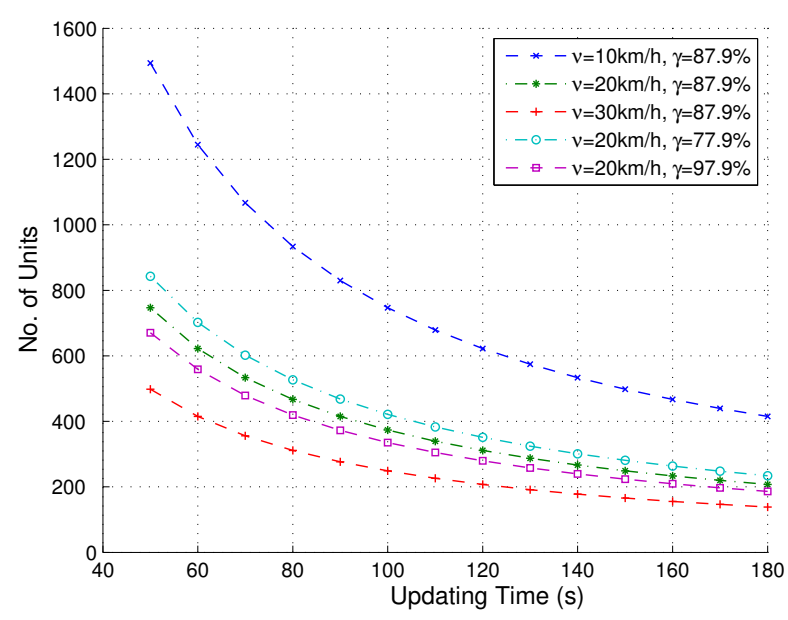

Fig. 11: Number of units against updating time

The number of 132 mobile sensing units is significantly lower than the 12000 sensors deployed for 8000 parking spaces. Fig. 11 illustrates how many mobile sensing units are needed as a function of the updating time (i.e. $1 / \tau$ ), cruising speed $\nu$, and the vehicle detection accuracy $\gamma$.

It is to be noted that, the simple estimation model presented is indifferent to the number and spatial distribution of parking bays, as all points of the roads will be covered by a $\nu \cdot \frac{1}{\tau}$ rolling window with frequency $\tau$ and accuracy $\gamma$. InfoRank [21] and CarRank [22] are two approaches that can provide a more in depth modelling and analysis on how to optimally find the appropriate vehicles for urban crowd sensing to cover different streets at different times and locations.

In the case of Guildford, with a city center size of approximately $5 \mathrm{~km}^{2}$, about 30 vehicles will be required. This can be achieved by equipping buses and taxis with the mobile sensing equipment or by creating a crowdsourcing platform to recruit private car owners. The crowdsourcing approach refers to the idea that vehicles equipped with our space detection system are sampling the road occupancy and contributing their individual views to the whole system while they are cruising the roads.

There is the question of how many drivers will agree to provide data, or even simply use and benefit from such a system. To the best of the authors knowledge no survey has been reported which indicates the propensity of vehicle owners to use their vehicles to monitor on-street parking and to share the data collected with the community. However, other related statistics may be relevant. For instance, over $14 \%$ of the US customers are interested in paying and acquiring a service specifically for real-time parking for their next vehicle [23]. The authors believe that if a car is purchased which is equipped with such a system, it is unlikely the purchaser would refuse it. In Europe, fuel efficiency (48\%) and congestion avoidance (39\%) are two of the features participants would be willing to buy a connected car for [24]. In the same study, $68 \%$ of the respondents are comfortable to share data for ecodriving support in real time. The mobile sensing system can be placed in these categories. In addition, $89 \%$ of respondents believe vehicle manufacturers should allow them to install third party telematic devices to their vehicles provided that they meet agreed security standards [25]. This can be an indication of the potential users if retrofitting older vehicle is an option.

The system can benefit not only the user but can also be seen as a community-based approach to help other users find a parking space faster and thus reduce overall congestion. As an example, the popular community-based navigation App, Waze, that allows users to share information about road and traffic conditions, has over 20,000 monthly active users in 38 countries and is used in over 185 countries [26]. These users can be considered as early adopters as they specifically open the App to use the services and have the community benefit from the information gathered as they drive. If such a service is to be embedded by default to vehicles, the number of active users can be expected to increase substantially.

\section{CONCLUSIONS}

In this article, a mobile space detection system has been described and tests have been carried out to compare the performance of the system to that of a fixed sensing system. The novelty of the presented mobile sensing unit is the map matching technique used to correct the GPS drifts/errors and the supervised learning algorithm to detect the cars/spaces. Based on the tests carried out in Guildford, measurements indicate that the overall accuracy of the mobile sensing system with map matching is $76 \%$ and it can reach $94 \%$ when the GPS accuracy is high. The GPS accuracy is an issue that can be solved by using more advanced localization techniques such as RTK GPS, DGPS, or Galileo system. The fixed sensor approach has a stable $85 \%$ accuracy rate for the tested scenario and is independent from any other technology, such as GPS.

The advantage of the mobile sensing approach is that it requires a significantly smaller number of sensor units compared with the fixed sensing solutions - 132 mobile sensing 
units compared to 12000 fixed sensors to cover 8000 parking spaces in the case of SFpark. The mobile sensing system becomes more pronounced as the number of parking spaces to be monitored increases. Initially, it is proposed that mobile sensing systems could be placed on vehicles such as buses, taxis or private cars to continually gather data as they travel along their routes. In the future, the mobile sensor system could also introduce a crowdsourcing approach where the public can install the sensors on their vehicles and make data collected while they travel available to the parking system, with appropriate reward in the form of reduced parking cost or simply free access to the parking applications.

In terms of future direction and work, lane-changing detection, a balanced guidance algorithm, precise vehicle localisation, and a right price model for on-street parking need to be considered in order to remove some of the limitations of the current system.

\section{REFERENCES}

[1] D. Shoup, "Cruising for parking," Transport Policy, vol. 13, no. 6, 2006.

[2] Garagescanner, "USharePark: smart secUres system to SHARE PARKing places in private garages." http://cordis.europa.eu/project/ren/ 199501 en.html, 2015. [Accessed 28 April 2017].

[3] E. Polycarpou, L. Lambrinos, and E. Protopapadakis, "Smart parking solutions for urban areas," in IEEE 14th International Symposium and Workshops on a World of Wireless, Mobile and Multimedia Networks (WoWMoM), pp. 1-6, 2013.

[4] S. Mahmud, G. Khan, M. Rahman, H. Zafar, et al., "A survey of intelligent car parking system," Journal of applied research and technology, vol. 11, no. 5 , pp. 714-726, 2013

[5] SFpark. www.sfpark.org, 2014. [Accessed 28 April 2017].

[6] Worldsensing, "FASTPRK: The easiest way to park." www.fastprk.com, 2015. [Accessed 28 April 2017].

[7] British Parking Association and Dr. Adam Snow, "Researching the research - executive summary." www.britishparking.co.uk, April 2015. [Accessed 26 April 2017].

[8] S. Mathur, T. Jin, N. Kasturirangan, J. Chandrasekaran, W. Xue, M. Gruteser, and W. Trappe, "Parknet: drive-by sensing of road-side parking statistics," in Proceedings of the 8th international conference on Mobile systems, applications, and services, pp. 123-136, ACM, 2010.

[9] S. Nawaz, C. Efstratiou, and C. Mascolo, "Parksense: A smartphone based sensing system for on-street parking," in Proceedings of the 19th annual international conference on Mobile computing \& networking, pp. 75-86, ACM, 2013.

[10] Z. Zhang, X. Li, H. Yuan, and F. Yu, "A street parking system using wireless sensor networks," International Journal of Distributed Sensor Networks, vol. 2013, 2013

[11] J. Lanza, L. Sánchez, V. Gutiérrez, J. A. Galache, J. R. Santana, P. Sotres, and L. Muñoz, "Smart city services over a future internet platform based on internet of things and cloud: The smart parking case," Energies, vol. 9, no. 9, p. 719, 2016

[12] Ethos V.O., "Case Study: Guildford." http://geomii.co/case-study-1guildford/, 2015. [Accessed 26 April 2017].

[13] Siemens, "Integrated Smart Parking Solution." www.mobility.siemens.com/mobility/global/en/urban-mobility/roadsolutions/integrated-smart-parking-solution/Pages/integrated-smartparking-solution.aspx, 2015. [Accessed 28 April 2017].

[14] Ford, "Parking Spotter." https://media.ford.com/content/fordmedia/ fna/us/en/news/2015/01/06/mobility-experiment-parking-spotteratlanta.html, 2015. [Accessed 28 April 2017].

[15] Smart Parking, "On-street parking." www.smartparking.com/on-street, 2015. [Accessed 28 April 2017]

[16] Smart Santander. www.smartsantander.eu/index.php/testbeds/item/132 santander-summary, 2015. [Accessed 28 April 2017].

[17] P. Ball, R. Liao, C. Roman, S. Ou, and E. Pow, "Analysis of fixed and mobile sensor systems for parking space detection," in Communication Systems, Networks and Digital Signal Processing (CSNDSP), 2016 10th International Symposium on, pp. 1-6, IEEE, 2016.

[18] G. M. Dias, B. Bellalta, and S. Oechsner, "Predicting occupancy trends in barcelona's bicycle service stations using open data," in SAI Intelligent Systems Conference (IntelliSys), pp. 439-445, IEEE, 2015.
[19] F. Marchal, "Trackmatching." https://mapmatching.3scale.net/, 2016. [Accessed 22 March 2017].

[20] P. Newson and J. Krumm, "Hidden markov map matching through noise and sparseness," in Proceedings of the 17th ACM SIGSPATIAL International Conference on Advances in Geographic Information Systems, GIS '09, (New York, NY, USA), pp. 336-343, ACM, 2009.

[21] J. A. Khan, Y. Ghamri-Doudane, and D. Botvich, "Autonomous identification and optimal selection of popular smart vehicles for urban sensing:an information-centric approach," IEEE Transactions on Vehicular Technology, vol. 65, pp. 9529-9541, Dec 2016.

[22] J. A. Khan and Y. Ghamri-Doudane, "Car rank: An informationcentric identification of important smart vehicles for urban sensing," in 2015 IEEE 14th International Symposium on Network Computing and Applications, pp. 184-191, September 2015

[23] Frost and Sullivan, "Strategic assessment of the smart parking market.” http://inrix.com/wp-content/uploads/2016/11/INRIX_ParkingStudy_Frost-and-Sullivan-Final-Report_052615.pdf, July 2015. [Accessed 24 November 2017]

[24] F. I. de l'Automobile Region I Europe The Middle East and Africa, "What europeans think about connected cars," February 2016.

[25] T. B. V. Rental and L. Association, "Bvrla fleet technology survey 2017 (white paper)," tech. rep., The British Vehicle Rental and Leasing Association, July 2017.

[26] Waze, "Driver satisfaction index." https://inbox-static.waze.com/ driverindex.pdf, 2016. [Accessed 24 November 2017].

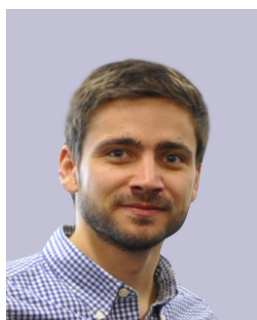

Cristian Roman is a postdoctoral researcher in the Communications Research Group at Oxford Brookes University. He holds a Ph.D. degree obtained from the same university in 2016, a M.Sc. in computer science from Supinfo, Paris, France and a M.Sc. degree in Broadband Networks from Oxford Brookes University, UK. His areas of interest includes mobile sensor systems and the design of scheduling algorithms for heterogeneous wireless communications for use in vehicular networks.

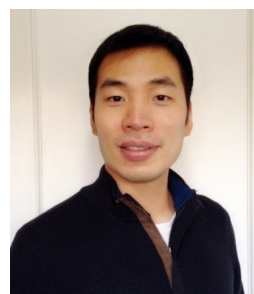

Ruizhi Liao holds a Ph.D. degree from the Department of Information and Communication Technology, Universitat Pompeu Fabra, Spain. His research interests include IEEE 802.11 amendments for next generation networks, MAC protocol designs, smart cities and intelligent transportation system. $\mathrm{He}$ is currently a Lecturer at The Chinese University of Hong Kong (Shenzhen), China

Peter Ball had over 20 years of experience in the telecommunications industry before joining Oxford Brookes University in 2003. He is currently a Reader in the department of Computing and Communication Technologies. His research activities include wireless communications for vehicular networks and wireless sensor networks for intelligent transport systems.

Shumao Ou is a member of IEEE. He received his Ph.D. degree in Electronic Systems Engineering and M.Sc. degree (with distinction) in Computer Information Networks from University of Essex, UK, in 2004 and 2007 respectively. From the end of 2006 to 2009, he worked in the School of Computer Science and Electronic Engineering, University of Essex as a Senior Research Officer. He is currently a senior lecturer at Oxford Brookes University.

Martin de Heaver leads GEOmii Technologies, developing parking, retail footfall and active mobility products. Martin has extensive experience in the design and delivery of major transportation technology projects in the road, rail and aviation sections, and is a Visiting Senior Research Fellow at King's College London, UK. 